\title{
Effects of RNA silencing of matrix metalloproteinase-2 on the growth of esophageal carcinoma cells in vivo
}

\author{
YU-GUANG SHEN $^{1 *}$, WEN FENG ${ }^{2 *}$, YI-JUN XU ${ }^{3 *}$, NA-NA JIAO $^{4}$, DA-QIANG SUN ${ }^{3}$, \\ WEN-DONG QU ${ }^{4}$, QUAN TANG $^{4}$, WEI XIONG ${ }^{4}$, YANG TANG $^{4}, \mathrm{YU} \mathrm{XIA}^{4}$, QING-YONG CAI ${ }^{4}$, \\ DA-XING LIU ${ }^{4}$, XUN ZHANG ${ }^{3}$, GANG XU $^{4}$ and GUI-YOU LIANG ${ }^{4}$ \\ ${ }^{1}$ Department of Thoracic and Cardiovascular Surgery, The First People's Hospital of Zunyi, Zunyi, Guizhou 563003; \\ ${ }^{2}$ Department of Pathology, Henan Tumor Hospital of Zhengzhou University, Zhengzhou, Henan 450000; \\ ${ }^{3}$ Thoracic Department, Tianjin Chest Hospital, Tianjin 300051; \\ ${ }^{4}$ Department of Thoracic and Cardiovascular Surgery, Affiliated Hospital of Zunyi Medical College, \\ Guizhou 563003, P.R. China
}

Received May 6, 2015; Accepted September 22, 2016

DOI: $10.3892 / \mathrm{ol} .2016 .5542$

\begin{abstract}
Esophageal carcinoma is one of the most common malignancies in China. Previous studies reported that matrix metalloproteinases (MMPs) have important roles in the progression and invasion of numerous types of solid tumors. Among the MMPs, MMP-2 has been closely associated with tumor growth and invasion. In the present study, a short hairpin RNA (shRNA) lentiviral expression vector targeting the MMP-2 gene was constructed in order to observe the inhibitory effect of MMP-2 gene silencing on the growth of the KYSE150 esophageal carcinoma cell line in vivo. Three small hairpin RNA sequences targeting MMP-2 were designed and cloned into lentiviral vectors. Following transfection of the lentiviral vectors into KTSE150 cells, MMP-2 mRNA and protein expression levels were examined by reverse transcription-quantitative polymerase chain reaction and western blotting, and the growth rate of cells was analyzed by MTT assays. Subsequently, tumor growth was assessed in nude mice. Lentivirus-mediated RNA interference effectively inhibited the expression of MMP-2 mRNA and protein in KYSE150 esophageal carcinoma cells, and suppressed the growth of esophageal carcinoma cells in vivo. The results of the present study suggested that lentivirus-mediated gene therapy targeting MMP-2 may be an attractive strategy for the treatment of esophageal
\end{abstract}

Correspondence to: Professor Xun Zhang, Thoracic Department, Tianjin Chest Hospital, Xi'an Road, Tianjin 300051, P.R. China E-mail: zhangxuntj@126.com

*Contributed equally

Key words: matrix metalloproteinase-2, esophageal carcinoma, RNA interference, lentivirus carcinoma and justifies the performance of further studies on the application of lentivirus vectors to cancer gene therapy.

\section{Introduction}

Esophageal carcinoma is one of the most common malignant cancers in China. As a result of extensive local cancer invasion, lymph node involvement and distant metastasis at the time of diagnosis, patients with esophageal carcinoma typically show rapidcancerprogressionandapoorprognosis(1).Ithaspreviously been demonstrated that the development of esophageal carcinoma is a complex, multi-step process involving a multitude of enzymes (2).

Human matrix metalloproteinases (MMPs) are a group of endopeptidases that degrade various components of the extracellular matrix (ECM) (3). MMPs have been shown to have important roles in tumor metastasis, invasion and angiogenesis (4). MMP-2, which is also known as type IV collagenase and gelatinase A, is a member of the MMP family that is located on the long arm of chromosome $16(16 q)$, is comprised of 13 exons and 12 introns, and has a molecular weight of $72 \mathrm{kDa}$ (5). MMP-2 degrades type IV collagen within basement membranes, which are the primary barrier to cancer invasion (6). Previous studies have reported a role for MMP-2 in the invasion of pancreatic, ovarian and lung cancer (7-9). However, the function of MMP-2 in esophageal carcinoma remains uncertain.

RNA interference (RNAi) using small interfering (si)RNAs to inhibit the expression of specific genes is a powerful and promising technology for basic research and therapeutic intervention (10-13). Our previous study demonstrated that MMP-2 knockdown using synthesized oligonucleotides inhibited the invasion and migration of the KYSE150 esophageal carcinoma cell line in vitro (14). In the present study, lentiviral vectors targeting the MMP-2 gene were constructed and transfected into KYSE150 cells, in order to observe the inhibitory effect of MMP-2 silencing on the growth of esophageal carcinoma cells in nude mice. The present study aimed to further clarify the 
Table I. Three hairpin DNA oligonucleotide sequences targeting matrix metalloproteinase-2 mRNA.

\begin{tabular}{ll}
\hline shRNAs & Sequences \\
\hline shRNA-1 & Sense: 5'-TGCGACAAGAAGTATGGCTTCTTTCAAG \\
& AGAAGAAGCCATACTTCTTGTCGCTTTTTC-3' \\
& Antisense: 5'-TCGAGAAAAAAGCGACAAGAAGTATGGCTT \\
shRNA-2 & CTTCTCTTGAAAGAAGCCATACTTCTTGTCGCA-3' \\
& Sense: 5'-TGGAGATACAATGAGGTGAAGATTC \\
& AAGAGATCTTCACCTCATTGTATCTCCTTTTTC-3' \\
shRNA-3 & Antisense: 5'-TCGAGAAAAAAGGAGATACAATGAGGT \\
& GAAGATCTCTTGAATCTTCACCTCATTGTATCTCCA-3' \\
& Sense: 5'-TGCAAACAGGACATTGTATTTGTTCAAGA \\
Non-targeting & GACAAATACAATGTCCTGTTTGCTTTTTC-3' \\
control & Antisense: 5'-TCGAGAAAAAAGCAAACAGGACATTGTATT \\
& TGTCTCTTGAACAAATACAATGTCCTGTTTGCA-3' \\
& Sense: 5'-TGTAGCGACTAAACACATCAATTCAAG \\
& AGATTGATGTGTTTAGTCGCATTCTTTTTC-3' \\
& Antisense: 5'-TCGAGAAAAAATAGCGACTAAACACATCAA \\
& TCTCTTGAATTGATGTGTTTAGTCGCATGCA-3'
\end{tabular}

shRNA, small hairpin RNA.

role of MMP-2 in esophageal carcinoma in vivo and to provide experimental evidence for pre-clinical gene therapy for esophageal carcinoma.

\section{Materials and methods}

Cell culture. The human embryonic kidney $293 \mathrm{~T}$ packaging cell line and KYSE150 esophageal carcinoma cell line were obtained from the Type Culture Collection of the Chinese Academy of Sciences (Shanghai, China) and cultured in RPMI-1640 medium supplemented with $10 \%$ fetal bovine serum (Gibco; Thermo Fisher Scientific, Inc., Waltham, MA, USA) at $37^{\circ} \mathrm{C}$ in a humidified incubator containing $5 \% \mathrm{CO}_{2}$.

Lentivirus vector construction and transfection into KYSE150 cells. The lentiviral vector system is composed of four plasmids: The expression plasmid and three packaging vectors, including pMD2.g (VSVG), pRSV-REV and pMDLg/pRRE (Shanghai Telebio Biomedical, Co., Ltd., Shanghai, China). The human MMP-2 gene (Gen Bank ID: 4313, NM_001127891.1, NM_004530.4) interference sequence was obtained using small hairpin (sh)RNA analysis software (http://www.invitrogen.com/rnai), and a Basic Local Alignment Search Tool analysis of the NCBI database (http://blast.ncbi.nlm.nih.gov/Blast.cgi) confirmed that it had no homology with other genes. Three self-complementary hairpin DNA oligonucleotides and a negative control targeting MMP-2 mRNA were synthesized. The sequences are shown in Table I. Subsequently, the DNA oligonucleotides were cloned into the lentiviral vectors and they were confirmed by DNA sequencing.

Lentiviral vectors and packaging vectors were transfected into $293 \mathrm{~T}$ cells. Following transfection, the cells were cultured for $8 \mathrm{~h}$, after which the culture medium was exchanged with
Dulbecco's modified Eagle's medium (Gibco; Thermo Fisher Scientific, Inc.). After $48 \mathrm{~h}$, the supernatant containing the retroviral particles was collected and then concentrated by centrifugation at 4,000 $\mathrm{x} g$ and $4^{\circ} \mathrm{C}$. A total of $2 \times 10^{5} \mathrm{KYSE} 150$ cells/well were transduced with viral supernatants, and the transfection efficiency was detected directly by assessing the expression ratio of green fluorescent protein (GFP) by fluorescence microscopy. Stable cell lines were obtained after selection by culture in medium containing $100 \mu \mathrm{g} / \mathrm{ml}$ ampicillin for 18 days.

Reverse transcription-quantitative polymerase chain reaction (RT-qPCR). Total RNA was extracted from cultured cells using the RNA purification kit (Sigma-Aldrich; Merck Millipore, Darmstadt, Germany). DNase I was used to remove any contaminating DNA. Subsequently, RNA was reverse transcribed into cDNA. The primers for the PCR were designed using Primer Premier 3 software (Premier Biosoft International, Palo Alto, CA, USA) and were synthesized by Beijing SBS Genetech, Co., Ltd. (Beijing, China). PCR was performed using Quantitative RT-PCR ReadyMix (Sigma-Aldrich; Merck Millipore). The primers for MMP-2 were as follows: Forward, 5'-TCCAGAGGCAATGCAGTGGGG-3' and reverse, 5'-CAG CTCTCCTTGGGGCAGCCA-3'. Primers for $\beta$-actin were as follows: Forward, 5'-ACCACAGTCCATGCCATCAC-3' and reverse, 5'-TCCACCACCCTGTTGCTGTA-3'. The PCR conditions involved denaturing the DNA at $94^{\circ} \mathrm{C}$ for $3 \mathrm{~min}$, followed by 30 cycles of amplification: $94^{\circ} \mathrm{C}$ for $30 \mathrm{sec}, 55^{\circ} \mathrm{C}$ for $1 \mathrm{~min}, 72^{\circ} \mathrm{C}$ for $1 \mathrm{~min}$ and a final extension step at $72^{\circ} \mathrm{C}$ for $10 \mathrm{~min}$. The data were analyzed using the $2^{-\Delta \Delta \mathrm{Cq}}$ method (15).

Western blotting. The cells were washed with cold PBS and lysed in pre-cooled radioimmunoprecipitation assay buffer (Pierce; Thermo Fisher Scientific, Inc.) containing proteinase 

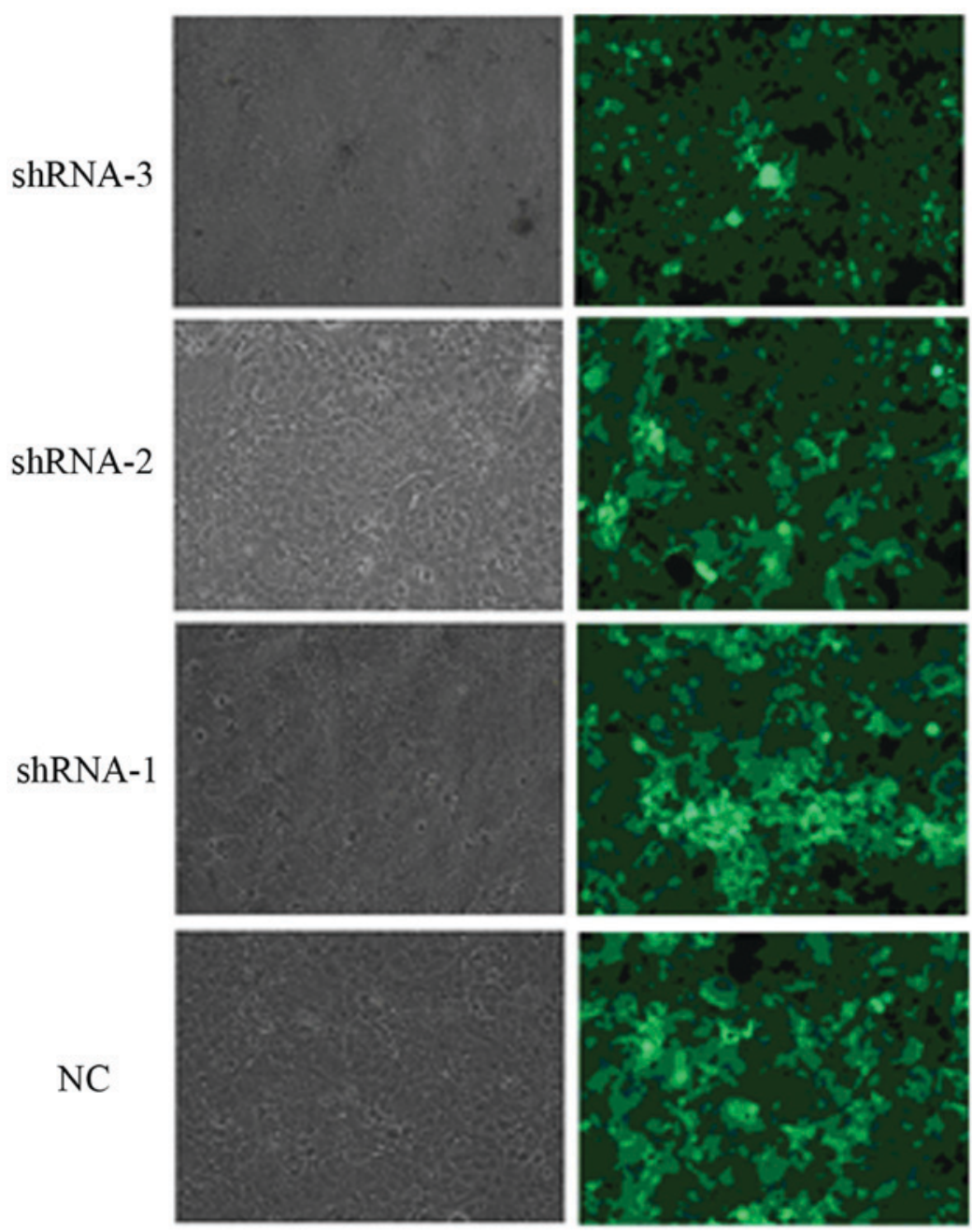

Light microscopy

Fluorescence microscopy

Figure 1. Micrographs of KYSE150 cells infected with recombinant lentiviruses. After being infected with the recombinant lentiviruses carrying the reporter GFP gene, the transfected cells expressed GFP proteins, as observed under a fluorescence microscope. The results indicated that $90 \%$ of KYSE150 cells were transfected with the recombinant lentiviruses. GFP, green fluorescent protein; shRNA, short hairpin RNA; NC, normal control.

inhibitors. The mixture was incubated for $30 \mathrm{~min}$ on ice, after which cell lysates were cleared of cell debris by centrifugation at $140,009 \times \mathrm{g}$ for $5 \mathrm{~min}$ at $4^{\circ} \mathrm{C}$. Protein concentrations were determined using the BCA protein assay kit (Pierce; Thermo Fisher Scientific, Inc.) according to the manufacturer's protocol. The samples were mixed in 59 loading buffer (Pierce; Thermo Fisher Scientific, Inc.), denatured at $96^{\circ} \mathrm{C}$ for $10 \mathrm{~min}$ and chilled on ice. Subsequently, equal amounts of protein $(60 \mu \mathrm{g})$ were separated by $15 \%$ SDS-PAGE and blotted onto nitrocellulose membranes. The membranes were stained with Ponceau Red (Sigma-Aldrich; Merck Millipore) to verify that the proteins had been transferred. Subsequently, membranes were blocked with 5\% nonfat milk and incubated with anti-MMP-2 (cat. no. HPA001939-100UL; 1:10,000 dilution; Sigma-Aldrich; Merck Millipore) and anti- $\beta$-actin (cat. no. CBL171; 1:10,000 dilution; Sigma-Aldrich; Merck Millipore) primary antibodies overnight at $4^{\circ} \mathrm{C}$. After washing with PBS, the membranes were incubated with horseradish peroxidase-conjugated secondary antibodies (1:5,000; cat. no., ZDR-5118; Zhongshan Golden Bridge Biotechnology Co., Ltd., Beijing, China) for $60 \mathrm{~min}$ at room temperature. Proteins were detected and quantified using the enhanced chemiluminescence detection system (ChemiDoc XRS System; Bio-Rad Laboratories, Inc., Hercules, CA, USA) and expression levels were normalized to $\beta$-actin

MTT assays. The viability of KYSE150 cells transfected with MMP-2-lentivirus and control lentivirus vectors, as well as blank control cells, were measured using MTT assays. Briefly, KYSE150 cells were infected with lentivirus in 6-well plates and re-seeded into 96-well plates at a density of 2,000 cells per well. After 1, 2, 3 or 4 days, the cells were treated with $20 \mu \mathrm{l}$ MTT solution $(5 \mathrm{mg} / \mathrm{ml})$ for $4 \mathrm{~h}$, after which the cell supernatants were removed and $150 \mu \mathrm{l}$ dimethyl sulfoxide was added to each well. After $15 \mathrm{~min}$, the optical density (OD) of each well was measured using a microplate reader with an absorbance wavelength of $570 \mathrm{~nm}$. All experiments were performed in triplicate.

In vivo experiments. A total of 24 female BALB/c nude mice (age, 6-8 weeks; weight, 18-22 g) were supplied by Beijing HFK Bioscience Co., Ltd. (Beijing, China). The mice were 


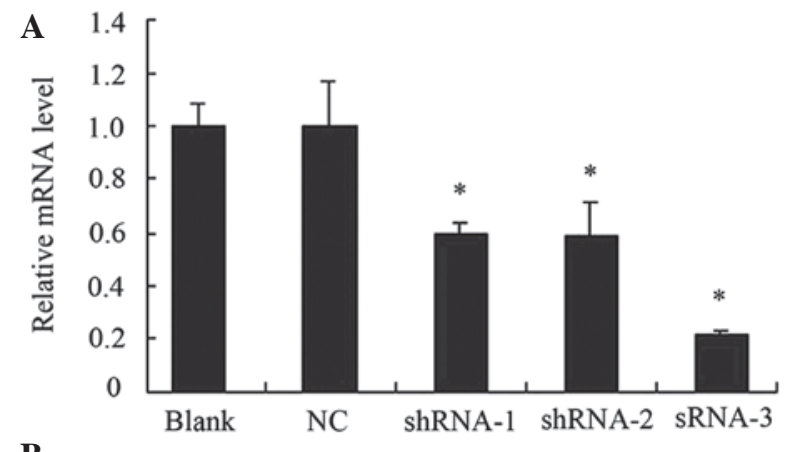

B
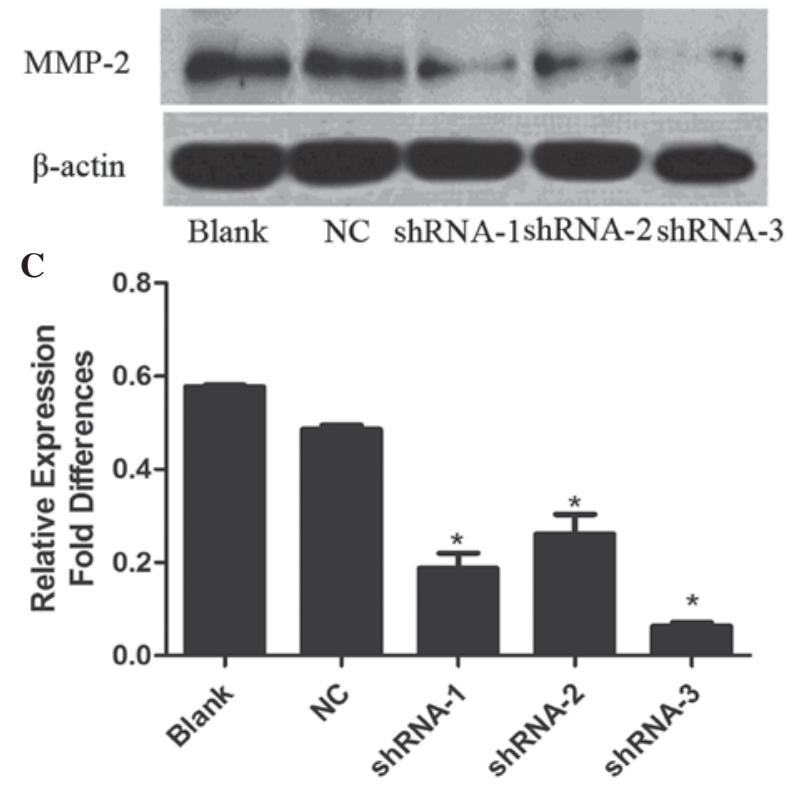

Figure 2. (A) Reverse transcription-quantitative polymerase chain reaction was used to determine the relative expression level of MMP-2 mRNA in five groups of KYSE150 cell lines. The mRNA expression levels of MMP-2 in the three groups transfected with MMP-2-specific shRNA were significantly downregulated ( $\mathrm{P}<0.05$ ), as compared with the blank group, while the NC cells showed no obvious change. (B) Western blotting demonstrated the downregulation of MMP-2 protein expression by shRNA in the KYSE150 esophageal cancer cell line. (C) Quantification of western blots. "P $<0.05$ vs. blank. MMP-2, matrix metalloproteinase-2; shRNA, short hairpin RNA; NC, normal control.

maintained under sterile conditions at $27^{\circ} \mathrm{C}$, exposed to $10 \mathrm{~h}$ light/dark cycles and fed a sterilized mouse diet and water ad libitum. Non-transfected control KYSE150 cells (blank group), MMP-2-non-targeting shRNA control (NC group) and MMP-2-shRNA-2-transfected KYSE150 cells $\left(2 \times 10^{6}\right.$ cells in $\left.0.1 \mathrm{ml}\right)$ were injected subcutaneously into the axilla of each BALB/c nude mouse. Tumor size was measured every 2 days in two perpendicular dimensions using vernier calipers and tumor volume was calculated according to the following formula: Tumor volume $\left(\mathrm{mm}^{3}\right)=1 / 2 \times\left(\mathrm{a} \mathrm{b} \mathrm{b}^{2}\right)$, where $\mathrm{a}$ and $\mathrm{b}$ refer to the longest and shortest dimensions, respectively.

Statistical analysis. All of the experimental data were analyzed using SPSS 13.0 software (SPSS, Inc., Chicago, IL, USA). Data are expressed as the mean \pm standard deviation. Statistical significance between the different groups was determined using the Student's t-test. $\mathrm{P}<0.05$ was considered statistically significant.

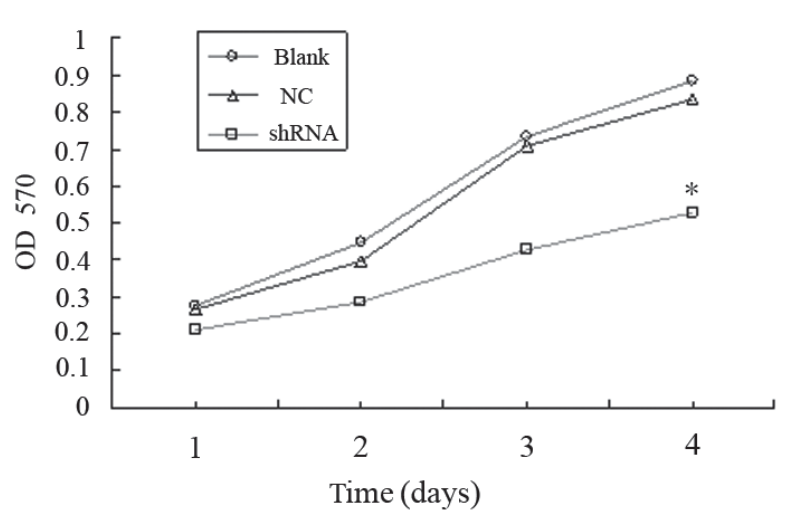

Figure 3. Viability of KYSE150 cell lines following lentivirus-mediated RNA interference of MMP-2, as assessed using MTT assays. KYSE-150 cells infected with lentiviruses carrying MMP-2-specific shRNA showed reduced cell viability, as compared with the cells in the blank and $\mathrm{NC}$ groups. ${ }^{*} \mathrm{P}<0.05$ vs. blank or NC group. MMP-2, matrix metalloproteinase-2; shRNA, short hairpin RNA; OD, optical density; NC, normal control.

\section{Results}

Transfection efficiency of lentiviral vectors in KYSE150 cells. The recombinant lentivirus vector was successfully constructed and confirmed by DNA sequencing. After being infected with the recombinant lentiviruses carrying the reporter GFP gene, the transfected KYSE150 cells were shown to express GFP, and the proportion of transfected cells was analyzed under a fluorescence microscope. The results indicated that $90 \%$ of KYSE150 cells were transfected with the recombinant lentiviruses (Fig. 1).

Lentivirus-mediated RNAi inhibits MMP-2 gene expression in KYSE150 cells. To evaluate the inhibition of MMP-2 mRNA expression, RT-qPCR was performed $72 \mathrm{~h}$ following transfection. The MMP-2 mRNA expression levels in shRNA-1-, shRNA-2- and shRNA-3-transfected KESY150 cells were reduced by $40.5,41.4$ and $78.9 \%$, respectively, as compared with the non-transfected control (blank group) ( $\mathrm{P}<0.05$; Fig. 2A). In addition, no significant difference was observed between the blank group and the MMP-2-non-targeting shRNA control (NC group) (Fig. 2A). The results indicated that the shRNA-3 oligonucleotide was the most effective for silencing of MMP-2 mRNA expression (Fig. 2A).

Lentivirus-mediated RNAi inhibits MMP-2 protein expression in KYSE150 cells. Western blotting demonstrated a significant reduction in MMP-2 protein expression in the KESY150 cells infected with shRNA-1 $(0.187 \pm 0.072$ of $\beta$-actin), shRNA-2 (0.261 \pm 0.095 of $\beta$-actin) and shRNA-3 (0.063 \pm 0.016 of $\beta$-actin), as compared with a non-targeting control $(0.486 \pm 0.021$ of $\beta$-actin) and blank control $(0.577 \pm 0.009$ of $\beta$-actin) $(\mathrm{P}<0.05$; Fig. $2 \mathrm{~B}$ and $\mathrm{C})$. These results indicated that shRNA-1, shRNA-2 and shRNA-3 all blocked MMP-2 expression, although shRNA-3 showed the most effective inhibition of MMP-2 expression. No obvious inhibition of MMP-2 protein was observed in the non-transfected control and blank control cells. 


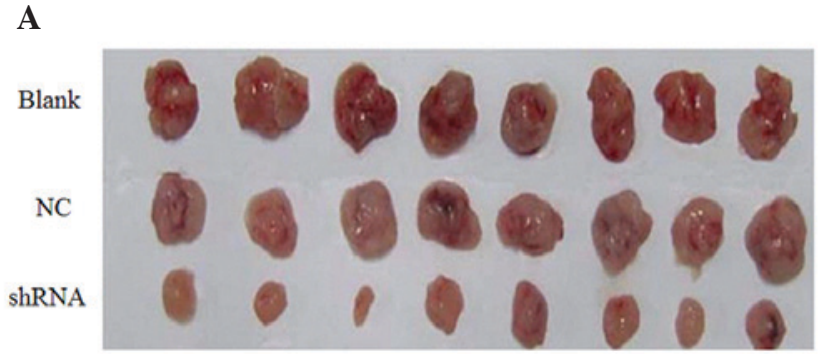

B

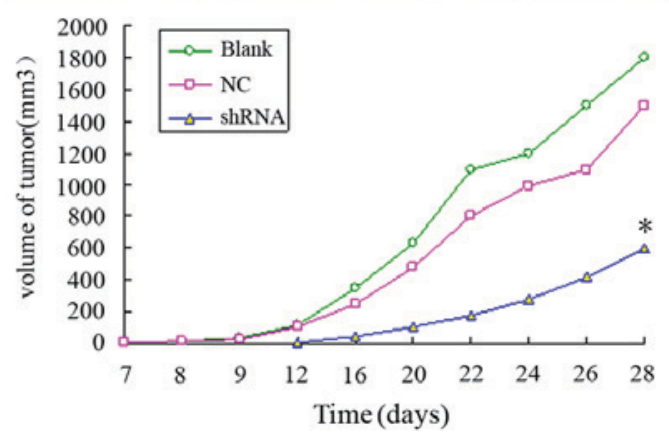

Figure 4. Lentivirus-mediated RNA interference targeting MMP-2 inhibits tumor growth in vivo. (A) Images of xenotransplant tumors. (B) The growth rates of MMP-2-knockdown nude mice tumors were significantly slower compared with those of blank control and NC groups ( $\mathrm{P}<0.05)$. MMP-2, matrix metalloproteinase-2; NC, normal control.

Lentivirus-mediated $R N A i$ against MMP-2 reduces KYSE150 cell viability in vitro. As the shRNA-3 oligonucleotide showed the most effective inhibition of MMP-2 expression, shRNA-3 was selected for MTT assays. As shown in Fig. 3, the viability of KYSE150 cells infected with lentivirus carrying shRNA-3 was significantly reduced, as compared with the NC and blank groups $(\mathrm{P}<0.05)$. These results suggest that knockdown of MMP-2 reduces the viability of KYSE150 cells in vitro.

Lentivirus-mediated RNAi against MMP-2 inhibits KYSE150 cell tumorigenicity in vivo. To determine whether lentivirus-mediated RNAi against MMP-2 was able to inhibit tumor development in vivo, nude mice were injected with MMP-2-shRNA3-infected KYSE150 cells, and the volume of the tumor was measured at 2-day intervals. As compared with the blank and NC groups, the MMP-2-shRNA3-infected KYSE150 cells resulted in significantly smaller tumors in the nude mice $(\mathrm{P}<0.05$; Fig. 4$)$, indicating that MMP-2 knockdown inhibited the tumorigenesis of esophageal cancer cells.

\section{Discussion}

Esophageal cancer was the fourth leading cause of cancer-associated mortality and the fifth most common diagnosed cancer in China in 2009, which was increased compared with the data from 2003-2007 (16). Although the results of surgery have improved significantly in recent years, the overall 5-year survival rates remain between 15 and $25 \%$ (17). Therefore, novel therapeutic strategies are urgently required for the treatment of this disease. Cancer metastasis is a multi-step process, and destruction or penetration of the basement membrane is thought to be critical for the successful metastasis of tumor cells (18). Human MMPs are thought to have important roles in tumor metastasis, invasion and angiogenesis (18).

Previous studies have reported a role for MMP-2 in the progression and invasion of numerous types of cancers $(19,20)$. In our previous study, it was demonstrated that MMP-2-knockdown using RNAi with synthesized oligonucleotides inhibited the invasion and migration of the KYSE150 esophageal carcinoma cell line in vitro (14). Furthermore, MMP-2 has exhibited a tumor-promoting function in many tumors in vivo, including ovarian (21), larynx (22) and brain (23) cancers. However, to the best of our knowledge, few studies have investigated the in vivo function of MMP-2 in esophageal cancer.

RNAi is a powerful means for post-transcriptional gene silencing and has been applied to a wide variety of eukaryotic organisms (24). Currently, chemically synthetic siRNAs are being evaluated for their use as highly-specific gene-silencing therapeutics, as well as their traditional role as an extremely powerful instrument for functional genomic analyses (25). However, there are several disadvantages associated with the use of synthesized siRNAs: i) The transduction of siRNA into cells usually leads to only transient silencing effects; ii) the transfection efficiency of siRNA may influence the silencing effects in target cells; and iii) transfected siRNA is expensive, as they must be chemically or enzymatically synthesized (26). To overcome these shortcomings, a stable RNAi DNA vector-based method has been developed (27-29). Approximately $70 \%$ of the vectors used in gene therapy clinical trials are represented by viral-based delivery systems (30). However, a number of failures with regard to gene therapy have been observed and thus, further optimization is required for the safe use of these vectors for clinical proposes in the future (31).

In the present study, a shRNA-lentiviral expression vector was used to obtain efficient knockdown of the MMP-2 gene in KYSE150 cells. The results showed that all shRNAs targeting MMP-2, including shRNA-1, shRNA-2 and shRNA-3, effectively inhibited the expression of MMP-2 mRNA and protein in KYSE150 cells, while the non-transfected and blank control groups showed no difference in MMP-2 expression. Notably, shRNA-3 was the most effective at suppressing MMP-2 expression and, therefore, it was selected for further investigation. Subsequently, it was demonstrated that MMP-2-shRNA-3 reduced the viability of KYSE150 esophageal carcinoma cells in vitro and tumorigenesis in vivo, as compared with the NC and blank control groups.

In conclusion, the results of the present study suggested that MMP-2 is a feasible RNAi target gene for esophageal carcinoma and that stable lentivirus-mediated shRNA targeting MMP-2 may be a promising and novel approach to the treatment of MMP-2-positive esophageal carcinoma. However, to further promote the application of this technique as a therapeutic approach, an effective and safe protocol should be developed.

\section{Acknowledgements}

The authors of the present study would like to thank Yong-Min Mao and Li-Li Zhao of the Tianjin Cardiovascular Institute for their technical assistance. 


\section{References}

1. Mukherjee S, Roth MJ,Dawsey SM, Yan W, Rodriguez-Canales J, Erickson HS, Hu N, Goldstein AM, Taylor PR, Richardson AM, et al: Increased matrix metalloproteinase activation in esophageal squamous cell carcinoma. J Transl Med 8: 91, 2010.

2. Li Y, Ma J, Guo Q, Duan F, Tang F, Zheng P, Zhao Z and Lu G: Overexpression of MMP-2 and MMP-9 in esophageal squamous cell carcinoma. Dis Esophagus 22: 664-667, 2009.

3. Poincloux R, Lizárraga F and Chavrier P: Matrix invasion by tumour cells: A focus on MT1-MMP trafficking to invadopodia. J Cell Sci 122: 3015-3024, 2009.

4. Yadav L, Puri N, Rastogi V, Satpute P, Ahmad R and Kaur G: Matrix metalloproteinases and cancer-roles in threat and therapy. Asian Pac J Cancer Prev 15: 1085-1091, 2014.

5. Price SJ, Greaves DR and Watkins H: Identification of novel, functional genetic variants in the human matrix metalloproteinase-2 gene: Role of Sp1 in allele-specific transcriptional regulation. J Biol Chem 276: 7549-7558, 2001.

6. Vihinen P and Kähäri VM: Matrix metalloproteinases in cancer: Prognostic markers and therapeutic targets. Int J Cancer 99: 157-166, 2002

7. Ellenrieder V, Alber B, Lacher U, Hendler SF, Menke A, Boeck W, Wagner M, Wilda M, Friess H, Büchler M, et al: Role of MT-MMPs and MMP-2 in pancreatic cancer progression. Int J Cancer 85: 14-20, 2000

8. Wang Y, Hu C, Dong R, Huang X and Qiu H: Platelet-derived growth factor-D promotes ovarian cancer invasion by regulating matrix metalloproteinases 2 and 9. Asian Pac J Cancer Prev 12: 3367-3370, 2011

9. Li GH, Cui YS, Wu QY, Zhang XJ and Gao YF: Clinicopathologic significance of $\beta$-catenin and matrix metalloproteinase-2 expression in non-small cell lung cancer. Med Oncol 30: 437, 2013.

10. Izquierdo M: Short interfering RNAs as a tool for cancer gene therapy. Cancer Gene Ther 12: 217-227, 2005.

11. Pushparaj PN, Aarthi JJ, Manikandan J and Kumar SD: SiRNA, miRNA and shRNA: In vivo applications. J Dent Res 87: 992-1003, 2008

12. Amarzguioui M, Rossi JJ and Kim D: Approaches for chemically synthesized siRNA and vector-mediated RNAi. FEBS Lett 579 : 5974-5981, 2005.

13. Li CX, Parker A, Menocal E, Xiang S, Borodyansky L and Fruehauf JH: Delivery of RNA interference. Cell Cycle 5: 2103-2109, 2006

14. Shen YG, Xu YJ, Shi ZL, Han HL, Sun DQ and Zhang X: Effects of RNAi-mediated matrix metalloproteinase-2 gene silencing on the invasiveness and adhesion of esophageal carcinoma cells, KYSE150. Dig Dis Sci 57: 32-37, 2012.

15. Livak KJ and Schmittgen TD: Analysis of relative gene expression data using real-time quantitative PCR and the 2(-Delta Delta C(T)) Method. Methods 25: 402-408, 2001.
16. Chen W, He Y, Zheng R, Zhang S, Zeng H, Zou X and He J: Esophageal cancer incidence and mortality in China, 2009. J Thorac Dis 5: 19-26, 2013

17. Pennathur A, Gibson MK, Jobe BA and Luketich JD: Oesophageal carcinoma. Lancet 381: 400-412, 2013.

18. Roy R, Yang J and Moses MA: Matrix metalloproteinases as novel biomarkers and potential therapeutic targets in human cancer. J Clin Oncol 27: 5287-5297, 2009.

19. Gong J, Zhu S, Zhang Y and Wang J: Interplay of VEGFa and MMP2 regulates invasion of glioblastoma. Tumour Biol 34: 11879-11885, 2014.

20. Honkavuori-Toivola M, Santala M, Soini Y, TurpeenniemiHujanen $\mathrm{T}$ and Talvensaari-Mattila A: Combination of strong MMP-2 and weak TIMP-2 immunostainings is a significant prognostic factor in endometrial carcinoma. Dis Markers 35: 261-266, 2013.

21. Seo JM, Park S and Kim JH: Leukotriene B4 receptor-2 promotes invasiveness and metastasis of ovarian cancer cells through signal transducer and activator of transcription 3 (STAT3)-dependent up-regulation of matrix metalloproteinase 2. J Biol Chem 287: 13840-13849, 2012.

22. Sun Y, Liu M, Yang B, Li B and Lu J: Role of siRNA silencing of MMP-2 gene on invasion and growth of laryngeal squamous cell carcinoma. Eur Arch Otorhinolaryngol 265: 1385-1391, 2008.

23. Maddirela DR, Kesanakurti D, Gujrati M and Rao JS: MMP-2 suppression abrogates irradiation-induced microtubule formation in endothelial cells by inhibiting $\alpha \mathrm{v} \beta 3$-mediated SDF-1/CXCR4 signaling. Int J Oncol 42: 1279-1288, 2013.

24. Zamore PD: RNA interference: Listening to the sound of silence. Nat Struct Biol 8: 746-750, 2001.

25. Leng Q, Woodle MC, Lu PY and Mixson AJ: Advances in systemic siRNA delivery. Drugs Future 34: 721, 2009.

26. Lin CC, Hsu JT, Huang KL, Tang HK and Lai YK: Stable RNA interference in Spodoptera frugiperda cells by a DNA vector-based method. Biotechnol Lett 28: 271-277, 2006.

27. Manjunath N, Wu H, Subramanya S and Shankar P: Lentiviral delivery of short hairpin RNAs. Adv Drug Deliv Rev 61: 732-745, 2009.

28. Dykxhoorn DM, Novina CD and Sharp PA: Killing the messenger: Short RNAs that silence gene expression. Nat Rev Mol Cell Biol 4: 457-467, 2003.

29. Lin CC, Hsu JT, Huang KL, Tang HK and Lai YK: Stable RNA interference in Spodoptera frugiperda cells by a DNA vector-based method. Biotechnol Lett 28: 271-277, 2006.

30. Ginn SL, Alexander IE, Edelstein ML, Abedi MR and Wixon J: Gene therapy clinical trials worldwide to 2012 - an update. J Gene Med 15: 65-77, 2013.

31. Chira S, Jackson CS, Oprea I, Ozturk F, Pepper MS, Diaconu I, Braicu C, Raduly LZ, Calin GA and Berindan-Neagoe I: Progresses towards safe and efficient gene therapy vectors. Oncotarget 6: 30675-30703, 2015. 